\title{
Intersubject Differences in False Nonmatch Rates for a Fingerprint-Based Authentication System
}

\author{
Jeroen Breebaart, Ton Akkermans, and Emile Kelkboom \\ Philips Research, HTC 34 MS61, 5656 AE Eindhoven, The Netherlands \\ Correspondence should be addressed to Jeroen Breebaart, jeroen.breebaart@philips.com \\ Received 4 September 2008; Accepted 7 July 2009 \\ Recommended by Jonathon Phillips
}

\begin{abstract}
The intersubject dependencies of false nonmatch rates were investigated for a minutiae-based biometric authentication process using single enrollment and verification measurements. A large number of genuine comparison scores were subjected to statistical inference tests that indicated that the number of false nonmatches depends on the subject and finger under test. This result was also observed if subjects associated with failures to enroll were excluded from the test set. The majority of the population (about 90\%) showed a false nonmatch rate that was considerably smaller than the average false nonmatch rate of the complete population. The remaining $10 \%$ could be characterized as "goats" due to their relatively high probability for a false nonmatch. The image quality reported by the template extraction module only weakly correlated with the genuine comparison scores. When multiple verification attempts were investigated, only a limited benefit was observed for "goats," since the conditional probability for a false nonmatch given earlier nonsuccessful attempts increased with the number of attempts. These observations suggest that (1) there is a need for improved identification of "goats" during enrollment (e.g., using dedicated signal-driven analysis and classification methods and/or the use of multiple enrollment images) and (2) there should be alternative means for identity verification in the biometric system under test in case of two subsequent false nonmatches.
\end{abstract}

Copyright (C) 2009 Jeroen Breebaart et al. This is an open access article distributed under the Creative Commons Attribution License, which permits unrestricted use, distribution, and reproduction in any medium, provided the original work is properly cited.

\section{Introduction}

The use of biometric characteristics for identity verification has been described as security enhancement on top of something one has (e.g., a card) and/or something one knows (e.g., a password) in many publications. The main reason for biometrics-enhanced identity management in such two or three-factor authentication approach is to reduce the risk of identity theft by increasing the difficulty of impersonation. In less critical applications, biometrics have also been proposed as replacement for passwords. With the ever-increasing number of login codes, passwords, and personal identification numbers (PINs), there is a strong need to reduce the amount of information that individuals have to memorize. Biometrics could provide a convenient solution for this increasing memory burden.

The use of biometrics in forensic context exists for a very long time. Around 1880, Dr. Henry Faulds recognized the importance of fingerprints for identification. In the 1890s, Alphonse Bertillon, a French anthropologist and police desk clerk used multiple body measurements to identify convicted criminals. Later Richard Edward Henry of Scotland Yard started to use fingerprints for the same purpose. These early methods all employed manual measurement and comparison for identification. Only during the last few decades, automated biometric identity verification systems have been introduced and have been subject to extensive research. One of the leitmotifs in biometrics research is the verification performance, expressed in average performance characteristics such as false acceptance rates (FARs), false rejection rates (FRR), and equal error rates (EERs). In most applications, the FAR and FRR are subject to a tradeoff; by modifying a comparison threshold value, security (expressed by the FAR) can be enhanced at the expense of a decreased convenience (expressed by the FRR) and vice versa, resulting in a detection error tradeoff (DET) curve. Similarly, performance tests on a subpopulation (excluding failures to acquire or to enroll) are expressed in terms of false match rates (FMRs) and false nonmatch rates (FNMR). In many cases, a biometric verification performance is characterized 
by an FRR (or FNMR) at a specific FAR (or FMR) which is typically 0.01 or 0.001 . These performance measures heavily depend on the biometric modality, the sensor type, the type of processing, and the corpus that is being used. As examples, various systems for face and iris recognition report an FRR in the range of $0.005-0.05$ at an FAR of 0.001 [1-3]; however, significantly worse performance is also reported $[4,5]$. For fingerprints, best-in-class solutions typically provide an FRR of approximately 0.04 at an FAR of 0.001 and about 0.03 for an FAR of $0.01[6,7]$.

Although the DET curve provides very meaningful information on the average performance of a biometric verification system given a certain population or corpus, it does not describe possible intersubject dependencies in verification performance. Subjects of a biometric system have been categorized into "sheep," "goats," "lambs" and "wolves," depending on their average (intrasubject) genuine and imposter scores [8]. This menagerie has been extended with "worms," "chameleons," "phantoms," and "doves" [9]. There is evidence that a number of these types indeed exist for certain biometric modalities and corpora [8-11] although the presence of "goats" in fingerprint corpora seems subject to debate [12].

The "goats" represent subjects that are difficult to recognize. They account for a disproportionate share of false nonmatches. These false nonmatches may cause these subjects to experience the system as being "inconvenient" possibly resulting in a decreased trust in a certain application which may also have negative consequences for their trust in biometrics in general. As an example, it has been shown that the age band has an effect on the performance of a fingerprint-based biometric system [3, 13] which suggests that elderly people may more often be associated with "goats" than younger people.

Failures to enroll or acquire may also pose challenges on biometric verification systems with regard to convenience. Subjects may have unreliable or absent biometric characteristics or body parts. It has often been assumed that between one and three percent of the general public does not have suitable biometric characteristics (cf. [2, 4]). A further challenge is caused by subjects that refuse to enroll. Subjects have in particular circumstances the right to object against the processing of biometric data on compelling legitimate grounds such as privacy concerns [14]. Other concerns may comprise health effects induced by biometric measurements, hygiene issues, the risk of stolen body parts containing a biometric, or negative associations such as fingerprints and crime. Surveys held in the US between 2001 and 2005 indicated that about $6-10 \%$ of the Americans found the use of finger and hand scan biometrics for law enforcement and governmental applications not acceptable $[15,16]$. In the commercial sector, a similar proportion of the population found it not acceptable to use biometrics for credit card transactions or Automated Teller Machines (ATMs). In Europe, a majority of consumers (92\%) now believe that a fingerprint is more secure than a signature, and $84 \%$ believes that biometrics are more secure than Chip and PIN [17].

Summarizing, failure of correct authentication in a biometric system may be associated with subjects that do not want to enroll, that cannot enroll, or that experience problems during verification. It is rather obvious that subjects belonging to the first two groups require an alternative means for authentication that is not based on biometrics. For the third group, the "goats," the situation is somewhat more subtle. This type is not easily detectable during enrollment if only a single measurement is available. Depending on the application, the difficulty to detect "goats" in an initial stage may jeopardize the success of a biometric verification system. For example, consider the case of biometrics-enabled ATMs. With billions of ATM transactions per month, a typical false nonmatch rate of 0.01 will result in a tremendous number of complaints, help desk calls, and service costs. Hence an FNMR of 0.01 will most likely not be acceptable for such an application and it will be crucial to understand and to mitigate the risk of false nonmatches. In conventional ATMs based on PIN authentication, a subject has multiple (typically 3) authentication attempts to resolve problems related with erroneously entered PINs. It is of interest to investigate the effect of such multiple verification attempts in a biometric authentication scheme and its influence on the resulting FNMR and FMR.

\section{FNMR Analysis}

Subject dependencies of FNMR have been found for speaker recognition [8], face recognition [11], and fingerprint recognition $[9,10,12]$. However, it has been argued that "hardto-match" fingerprints are resulting from properties of a certain (low-quality) measurement, rather than resulting from individual biometric characteristics themselves [12]. Furthermore, although the existence of subject interdependencies has been shown by statistical inference tests, most studies do not provide a clear insight in the distribution of intersubject FNMRs.

2.1. Fingerprint Corpus. An analysis of intersubject FNMRs was carried out based on the Ministerio de Ciencia y Tecnología (MCYT) baseline fingerprint corpus [18]. This database contains 12 images of all 10 fingers from 330 subjects that were located in four different institutions. All combinations of image number, finger, and subject have been measured using two acquisition devices: one optical sensor (UareU from Digital Persona) and one capacitive sensor (model 100SC from Precise Biometrics). Both sensors were operating at a resolution of $500 \mathrm{dpi}$. All fingerprint capturing was accomplished by the supervision of an operator using three levels of control by the subject that differed in the amount of visual feedback with respect to finger placement provided on a computer screen. In a subjective quality assessment on a subset of the data, $5 \%$ of the images was found to be of very bad quality, $20 \%$ of low quality, $55 \%$ of medium quality, and $20 \%$ of high quality (see [18] for details).

The total amount of fingerprint images amounts thus $330 \times 10 \times 12 \times 2=79,200$ images. Since 12 measurements are available for each subject, finger, and sensor, the maximum number of unique genuine comparisons per subject, finger, and sensor equals 66 (under the assumption 
that for all images a suitable minutiae template could be established). Hence for each finger and sensor, $66 \times 330=$ 21780 genuine comparisons can in principle be obtained, resulting in a total number of genuine comparisons per sensor of 217800 , and 435600 in total.

All 79200 images were converted to minutiae templates using a state-of-the-art commercially available minutiaeextractor and comparator solution. The minutiae-extractor also provides image quality ratings; the corresponding comparator solution operates symmetrically, that is, a comparison score of A with B is equal to B with A. Some of the images could not be converted to minutiae templates either due to a failure to acquire or a failure to enroll. Since the employed solution does not indicate whether a failure was due to acquisition or enrollment difficulties, we will refer to such failures as failures to enroll in the remainder of this paper. For the capacitive sensor, the system could not enroll one image for one finger of one subject. For four other subjects, none of the images from any finger could be enrolled. Hence out of the 39.600 images, $1+4 \times 12 \times 10=$ 481 images resulted in a failure to enroll, corresponding to a failure to enroll rate in terms of the number of images of 0.0121 . For the optical sensor, 4 subjects could not be enrolled for one finger and one image. One subject could not enroll one image from two fingers. One subject could not enroll any image from any finger. Hence, in total, $4+$ $2+120=126$ images resulted in a failure to enroll, which corresponds to a rate of 0.0032 .

From these data, two databases containing comparison scores were constructed described as follows.

(1) A full database, containing all genuine comparison scores within the same sensor, resulting in 435600 genuine comparison scores in total (217 800 for each sensor). Comparisons that involved an image that caused a failure to enroll were set to a similarity score of zero to ensure a reject irrespective of the (positive) comparison threshold. The imposter comparisons comprised a subset of 792000 combinations. Tests on this database describe the FAR-FRR tradeoff (i.e., including the effect of failure to enroll).

(2) A balanced database containing only subjects for which all images could be enrolled. Hence the resulting database is fully balanced (i.e., the same number of fingers and measurements per finger for each subject). This process resulted in 214500 genuine comparisons for the capacitive sensor (325 subjects), and 213840 genuine comparisons for the optical sensor (324 subjects). The number of imposter comparisons amounted to 384000 and 381720 , for the capacitive and optical sensors, respectively. Performance tests on this database are more closely related to FMRs and FNMRs, while minimizing the effect of low-quality data that could result in failure to enroll.

2.2. DET Curves. Separate DET curves were constructed for the optical and capacitive sensors from the genuine and imposter comparison scores. The results are visualized
TABLE 1: Error rates for the two sensors and the two databases (the full database providing FRRs and the balanced database providing FNMRs).

\begin{tabular}{lcccl}
\hline Sensor & EER (full) & EER (bal) & FRR@0.001 (full) $\begin{array}{l}\text { FNMR@0.001 } \\
\text { (bal) }\end{array}$ \\
\hline Capacitive & 0.0240 & 0.0138 & 0.0295 & 0.0181 \\
Optical & 0.0064 & 0.0034 & 0.0075 & 0.0040 \\
\hline
\end{tabular}

in Figure 1 for the full database. The solid line represents the capacitive sensor, the dashed line represents the optical sensor. The EER for the capacitive sensor amounts to 0.024; the EER for the optical sensor amounts to 0.0064. At a FAR of 0.001 , the FRR for the capacitive and optical sensors amounts to 0.0295 and 0.0075 , respectively. As can be observed, the optical sensor performs significantly better than the capacitive sensor: across the full DET curve, the FRR for the optical sensor is almost 4 times smaller than the FRR of the capacitive sensor for the same FAR. These results confirm earlier statement on quality differences between optical and capacitive sensors [19]. A similar analysis was performed for the balanced database. A comparison between the full and balanced database error rates is provided in Table 1. As can be observed, the EERs and FNMRs for the balanced database are about twice as low as for the full database (FRRs).

2.3. Statistical Inferences. The existence of "goat-" like behavior is investigated using statistical inference tests. The data is tested to support the null-hypothesis that the genuine comparison scores do not depend on the subject or finger indices. A nonparametric (Kruskal-Wallis) test was employed on the genuine comparison scores from the balanced database. The Kruskal-Wallis test can only be employed to investigate one factor; hence, the test was performed four times to cover all combinations of the two sensors and the two effects under test (subject index and finger index). The results are provided in Table 2. All null hypotheses that the subject or finger index did not have any effect on the comparison scores are rejected based on the observed $\chi^{2}$ values. Hence, it is concluded that the false nonmatch rates are subject to "goat-" like behavior.

The comparison scores were also subjected to a twoway analysis of variance (including interaction) with the finger index and subject as main effects, and the comparison score as dependent variable. The resulting $F$ values and the corresponding probability of falsely rejecting the null hypothesis, that is, none of the effects or interactions is significant, are provided in the last two columns of Table 2. In line with the results obtained from the Kruskal-Wallis test, both factors and their interaction were found to have a significant effect on the comparison scores. The same analyses were also carried out on the full database which gave the same qualitative result.

2.4. Intersubject Distribution of FNMR. The presence of significant effects of subject and finger index on the comparison scores for both sensors does not provide any insight in the actual distribution of FNMRs across subjects or fingers. To investigate the range of FNMRs between subjects, 
TABLE 2: Results for the Kruskal-Wallis test and analysis of variance (ANOVA) test for the optical and capacitive sensors. The factors that were taken into account were the subject index and the finger index. Tests were performed on the balanced database.

\begin{tabular}{|c|c|c|c|c|c|c|}
\hline Sensor & Effect & $\mathrm{df}$ & $\chi^{2}$ & $p>\chi^{2}$ & $F$ & $p>F$ \\
\hline Capacitive & Subject & 324 & 68276.05 & 0 & 510.08 & 0 \\
\hline Capacitive & Finger & 9 & 5813.31 & 0 & 1621.18 & 0 \\
\hline Capacitive & Interaction & 2916 & $\mathrm{n} / \mathrm{a}$ & $\mathrm{n} / \mathrm{a}$ & 44.62 & 0 \\
\hline Optical & Subject & 323 & 66894.26 & 0 & 552.18 & 0 \\
\hline Optical & Finger & 9 & 17047.04 & 0 & 5116.24 & 0 \\
\hline Optical & Interaction & 2907 & $\mathrm{n} / \mathrm{a}$ & $\mathrm{n} / \mathrm{a}$ & 42.15 & 0 \\
\hline
\end{tabular}

the number of false nonmatches within the set of all 66 genuine comparisons was computed for a threshold value that resulted in a global FMR of 0.001. The threshold was determined separately for each of the two sensors to compensate for performance differences between the sensors and was carried out on the balanced database.

In the following, the number of false nonmatches at a false match rate $\phi_{i}$ within a set of $N$ genuine comparisons is given by $x_{i, j, k}\left[\phi_{i}\right]$ for sensor $i$, subject $j$, finger $k$. If one assumes that each of the $N$ genuine comparisons for a given sensor $i$ has a constant probability for a false nonmatch that only depends on the false match rate $\phi_{i}$, the expected number $\mu_{i}\left[\phi_{i}\right]$ of false nonmatches within a set of $N=66$ genuine comparisons would be given by

$$
\mu_{i}\left[\phi_{i}\right]=N \psi_{i}\left[\phi_{i}\right]
$$

with $\psi_{i}\left[\phi_{i}\right]$ the estimate of the probability of a false nonmatch $\psi_{i}\left[\phi_{i}\right]$ for a false match rate $\phi_{i}$, given by

$$
\psi_{i}\left[\phi_{i}\right]=\frac{\sum_{j} \sum_{k} x_{i, j, k}\left[\phi_{i}\right]}{J K N} .
$$

In the absence of any intersubject or finger index dependencies, the variable $x_{i, j, k}\left[\phi_{i}\right]$ is then expected to follow a binomial distribution with mean $N \psi_{i}\left[\phi_{i}\right]$ and variance $N \psi_{i}\left[\phi_{i}\right]\left(1-\psi_{i}\left[\phi_{i}\right]\right)$. This expected distribution is visualized in Figure 2 by the solid lines. Figure 2(a) represents the capacitive sensor; the lower panel represents the optical sensor. In both the upper and lower panels, the horizontal axes indicate the number of nonmatches (in 66 attempts), the vertical axes represent the population proportion. The numbers inbetween the upper and lower panels represent the FNMR corresponding to the number of false nonmatches in 66 attempts. The capacitive sensor (Figure 2(a)) has a maximum at one nonmatch out of 66 which corresponds to the FNMR of 0.0181 at an FMR of 0.001 that was also provided in Table 1. The optical sensor (Fihure 2(b)) has a maximum at zero nonmatches which is caused by the smaller overall FNMR of 0.0040 . The far-most right point on the curves represents 9 or more nonmatches out of 66 . For the capacitive sensor, the probability of finding 9 or more false nonmatches out of 66 according to the binomial distribution equals $3.01 e-6$; for the optical sensor this value equals to $7.7 e-12$ (not shown in the figure).

The observed FNMRs per subject based on an individual comparison threshold for each sensor to result in an overall

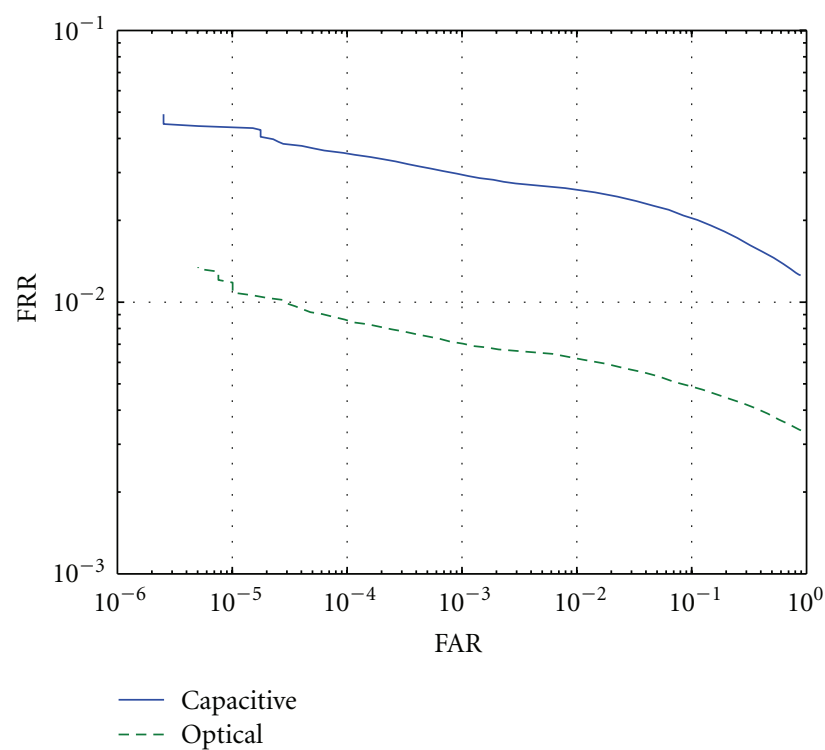

FIgure 1: DET curves for the capacitive (solid line) and optical (dashed line) sensors based on the full database (including failures to enroll).

FMR of 0.001 are given by the dashed lines in Figure 2. These curves represent the genuine comparisons for all subjects and fingers, that is, different fingers of one subject can be interpreted as additional subjects. All number of observations are normalized to sum to +1 to allow direct comparison with the binomial distribution given by the solid line. Interestingly, the curve for the observed number of false nonmatches is quite different from the binomial distributions, for both the capacitive and optical sensors. Two trends can be observed: (1) the number of subjects with zero false nonmatches is larger than expected based on a binomial distribution, and (2) the number of subjects with 9 or more false nonmatches is also significantly larger than expected. The proportion of subjects that obtained 9 or more false nonmatches (which corresponds to an FNMR of 0.136 or more) equals 0.0505 and 0.0145 , for the capacitive and optical sensors, respectively. The proportion of subjects with 23 or more nonmatches (an FNMR of 0.33 or larger) amounted 0.0120 and 0.0006 , for the capacitive and optical sensors. Hence, the observed frequencies of finding 23 or more nonmatches in a trial of 66 is 3 to 7 orders of magnitude larger than is expected based on a binomial distribution. 


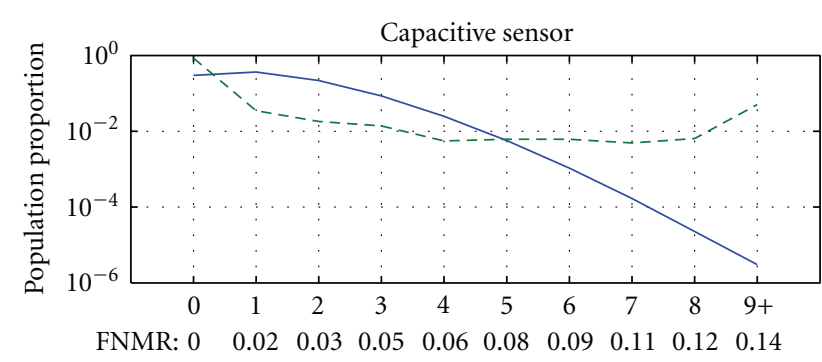

Number of false non-matches

(a)

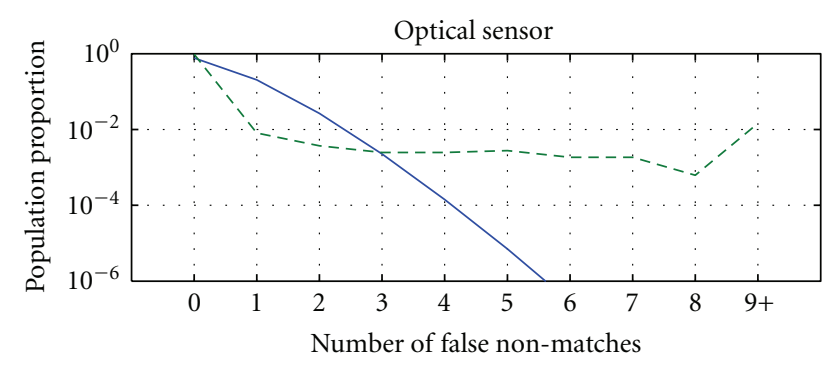

- Expected

- - - Observed

(b)

Figure 2: Distribution of the expected (solid lines) and observed (dashed lines) number of false nonmatches across subjects and fingers for the capacitive sensor (a) and the optical sensor (b). The numbers in between panels represent the corresponding false nonmatch rates.

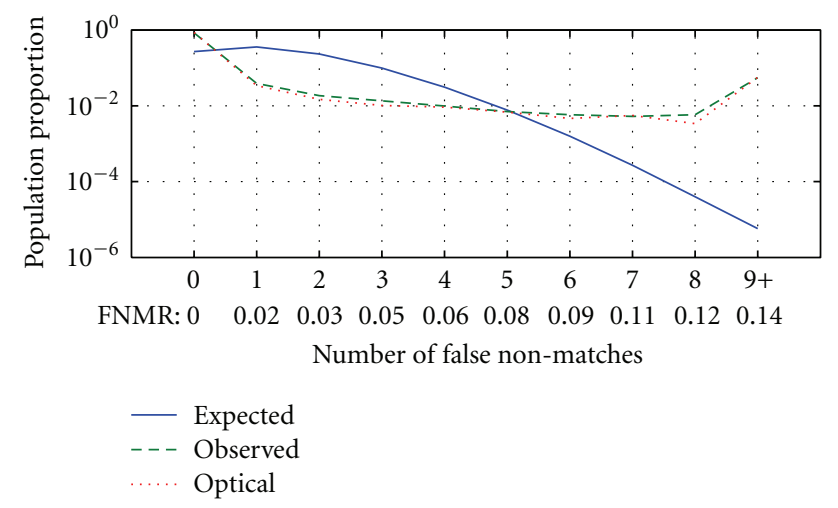

FIgURE 3: Distribution of expected (solid lines) and observed (dotted and dashed lines) number of false nonmatches across subjects for the capacitive sensor (dashed line) and the optical sensor (dotted line) based on a threshold to result in a mean FNMR of 0.02 .

One possible reason for finding a relatively large population of subjects with a high FNMR is that these could be resulting from "weak" fingers that more often causes nonmatches. To investigate the distribution of interclass FNMRs when excluding the effect of different FNMRs per finger, a separate comparison threshold was estimated for each finger index and sensor such that across all subjects, the FNMR was equal to a fixed value of 0.02 when measured for one finger and sensor across all subjects. The distribution of false nonmatches in a set of 66 attempts is shown in Figure 3. The expected values based on the binomial distribution with mean probability of 0.02 are given by the solid line; the observed distributions for the capacitive and optical sensors are shown by the dashed and dotted lines, respectively. Interestingly, using a separate threshold for each sensor and finger to result in the same mean FNMR, the observed distributions of FNMRs across subjects are very similar. Furthermore, there is a significant discrepancy between the expected (binomial) distribution and the observed distribution. More than 5\% of the population obtained 9 or more false nonmatches, which is significantly larger than the expected value of $5.8 e-6$. Another interesting observation is that for both sensors, about $90 \%$ of the subjects has an FNMR which is smaller than the population average of 0.02 , while only 10\% has an FNMR which is (significantly) larger.

2.5. Multiple Verification Attempts. If multiple verification attempts are allowed in a verification system, the expected number of false matches will typically increase if the comparison threshold is kept constant (e.g., assuming that an imposter will use a different finger during each attempt to maximize the false match probability). If the false match probability of the $n$th trial out of $N$ using sensor $i$ is assumed to be constant across subjects and fingers and given by $\phi_{i}[n, N]$, the probability that at least one of $N$ attempts will give a false match $\Phi_{i}[N]$ is given by

$$
\Phi_{i}[N]=1-\prod_{n=1}^{N}\left(1-\phi_{i}[n, N]\right) .
$$

If one also assumes that the probability $\phi_{i}[n, N]$ is independent of trial number $n$ and $\phi_{i}[n, N] \ll 1$, this can be approximated quite accurately by

$$
\Phi_{i}[N] \approx N \phi_{i}
$$

Said differently, the false match probability increases approximately linearly with the number of attempts if the comparison threshold is kept constant.

The number of false nonmatches will typically decrease with the increasing number of attempts. If the false nonmatch probability for attempt $n$ out of $N$ given by $\psi_{i}\left[\Phi_{i}[N], n, N\right]$, the probability that all $N$ attempts will result in, a false nonmatch is given by

$$
\Psi_{i}\left[\Phi_{i}[N], N\right]=\prod_{n=1}^{N}\left(\psi_{i}\left[\Phi_{i}[N], n, N\right]\right) .
$$

If one assumes the probabilities $\psi_{i}\left[\Phi_{i}[N], n, N\right]$ to be independent on trial $n$, this would result in

$$
\Psi_{i}\left[\Phi_{i}[N], N\right]=\psi_{i}\left[\phi_{i}\right]^{N} .
$$

Hence, an important consequence of the dependency of both FMR and FNMR on the number of attempts is that the comparison threshold should be dependent on the number of allowed attempts if a fixed FMR is desired. 


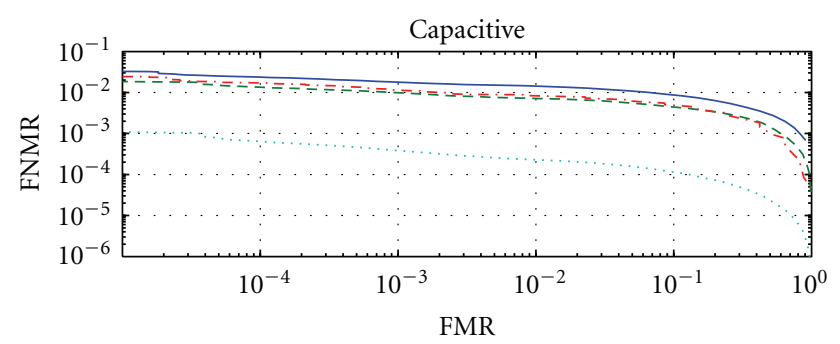

(a)

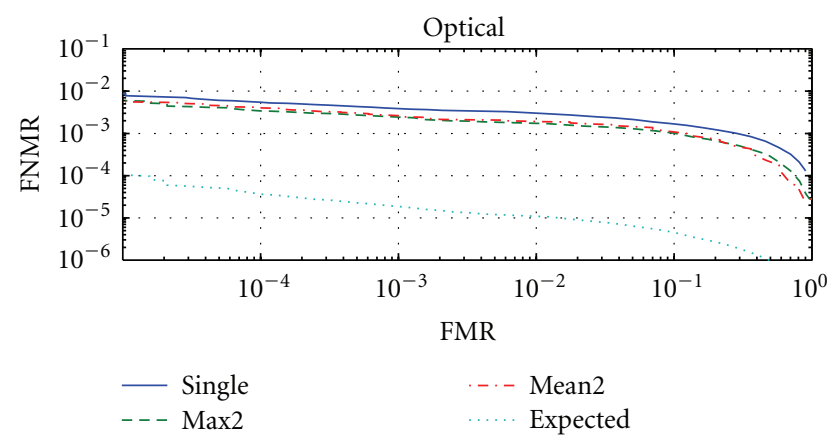

(b)

FIgURE 4: DET curve for the capacitive sensor (a) and the optical sensor (b) based on the balanced database. The solid line represents the FMR/FNMR tradeoff for a single attempt. The dashed lines represent the performance based on the maximum comparison score of 2 attempts ( $\max 2$ ); the dash-dotted lines represent the performance for the mean comparison score across 2 attempts (mean2). The dotted curve represents the expected FMR/FNMR tradeoff assuming constant false nonmatch and false match probabilities for each trial.

To investigate the effect of multiple verification attempts, a two and three trial case was simulated by taking the maximum comparison similarity score across two or three genuine comparisons, respectively. The same process was employed for the imposter scores using fingerprints from different subjects across the attempts. The resulting DET curves are visualized in Figures 4 and 5 for the balanced database. Figures 4(a) and 5(a) represent the capacitive sensor, Figures 4(b) and 5(b) represent the optical sensor. The solid, dashed, and dash-dotted lines represent a single trial, the maximum comparison, and the mean comparison scores across multiple attempts, respectively.

As can be observed from Figures 4 and 5, the possibility of multiple verification attempts has a positive influence on the verification performance. For the capacitive sensor, the FNMR of 0.018 at an FMR of 0.001 for a single verification attempt decreases to 0.011 and 0.010 for two attempts according to a "mean" and "max" rule, respectively. For the three-trial case, the respective FNMRs are equal to 0.0096 and 0.0075 . The optical sensor shows a similar trend. The FNMRs for a single trial at an FMR of 0.001 correspond to 0.0040 . For two attempts, the FNMRs are equal to 0.0026 and 0.0024 (for the "mean" and "max" rules, resp.). For three attempts, these rates are equal to 0.0020 and 0.0018 .

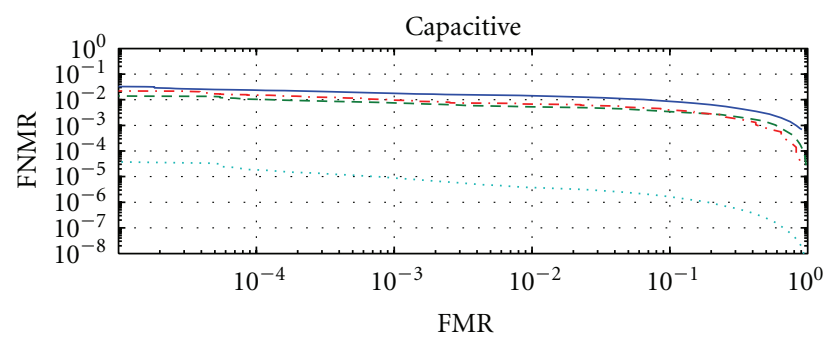

(a)

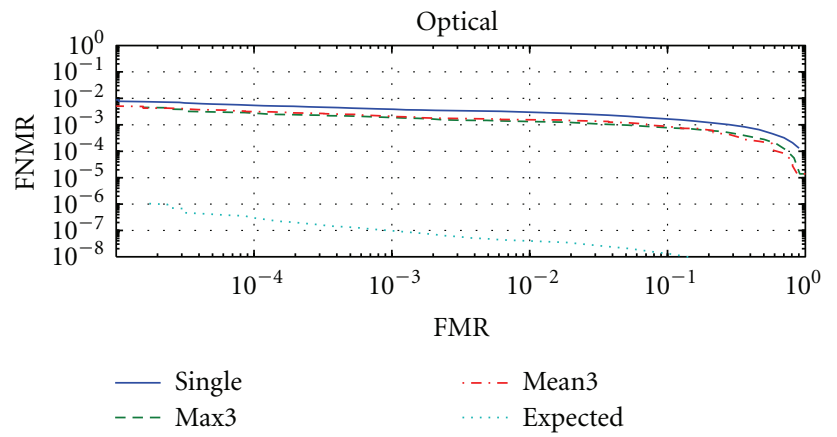

(b)

FIGURE 5: DET curve for the capacitive sensor (a) and the optical sensor (b) based on the balanced database. The solid line represents the FMR/FNMR tradeoff for a single attempt. The dashed lines represent the performance based on the maximum comparison score of 3 attempts $(\max 3)$; the dash-dotted lines represent the performance for the mean comparison score across 3 attempts (mean3). The dotted curve represents the expected FMR/FNMR tradeoff assuming constant false nonmatch and false match probabilities for each trial.

For both sensors, the "max" rule provides the lowest FNMR at a given FMR. The ratios of FNMRs at a fixed FMR of 0.001 for two attempts compared to one trial equal to 0.55 and 0.60 (for the capacitive and optical sensors, resp.). For three attempts, these ratios are equal to 0.42 and 0.45 , respectively, when compared to the single-attempt case. However, these improvements are significantly smaller than the expected DET curve based on the independence assumption of FNMR and FMR rates for each trial, which is represented by the dotted curves in Figures 4 and 5. This curve was created by transforming the single-attempt curve to a multiple-attempt curve using (6) and (3).

2.6. Discussion. When attempting to enroll the 79,200 images, the failure to enroll rate amounted about 0.012 for the capacitive and 0.003 for the optical sensors, respectively. For the capacitive sensor, the value of 0.012 is quite in line with the assumption that between 1 and $3 \%$ of a population has difficulties or failures to enroll. The value of 0.003 for the optical sensor is relatively low in this respect.

The DET curves based on the full database shown in Figure 1 indicate that the two sensors employed in the test differ considerably in terms of verification performance. 


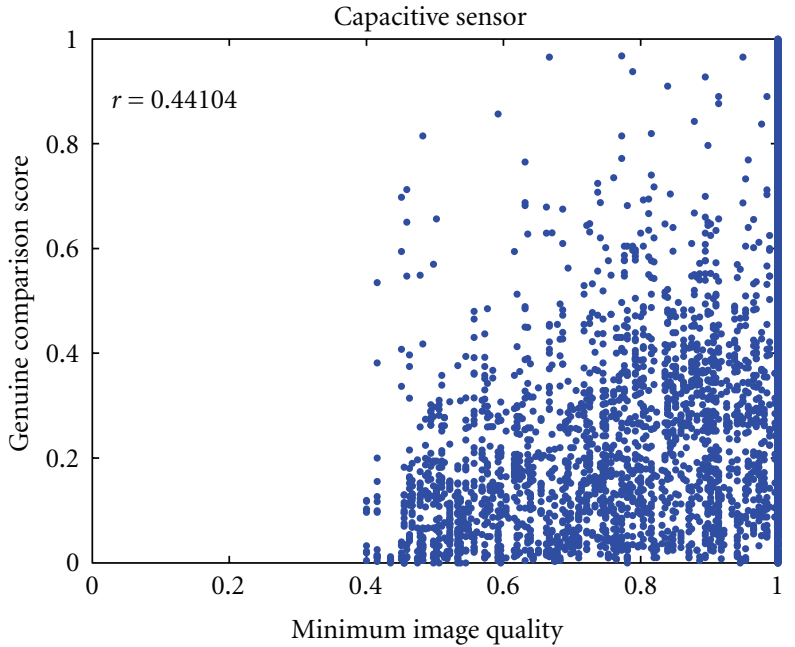

(a)

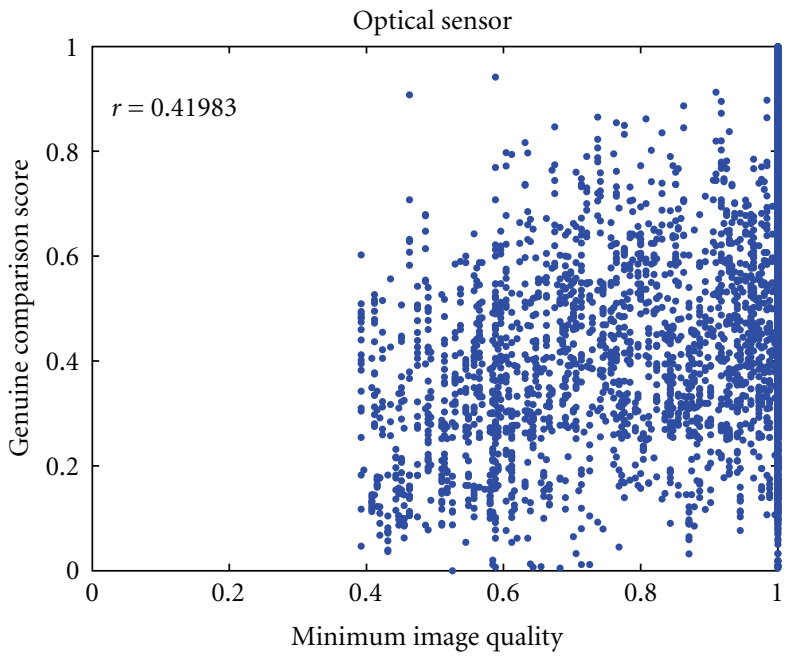

(c)

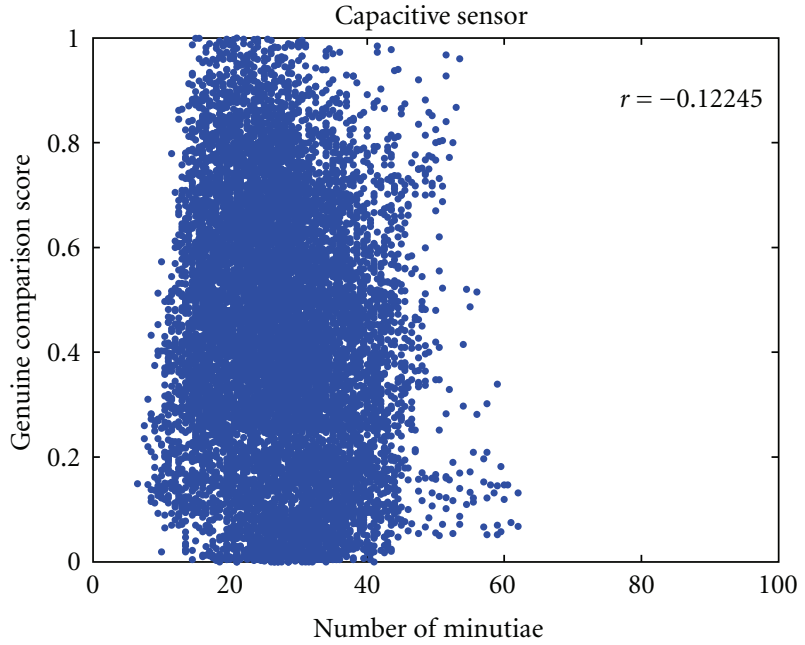

(b)

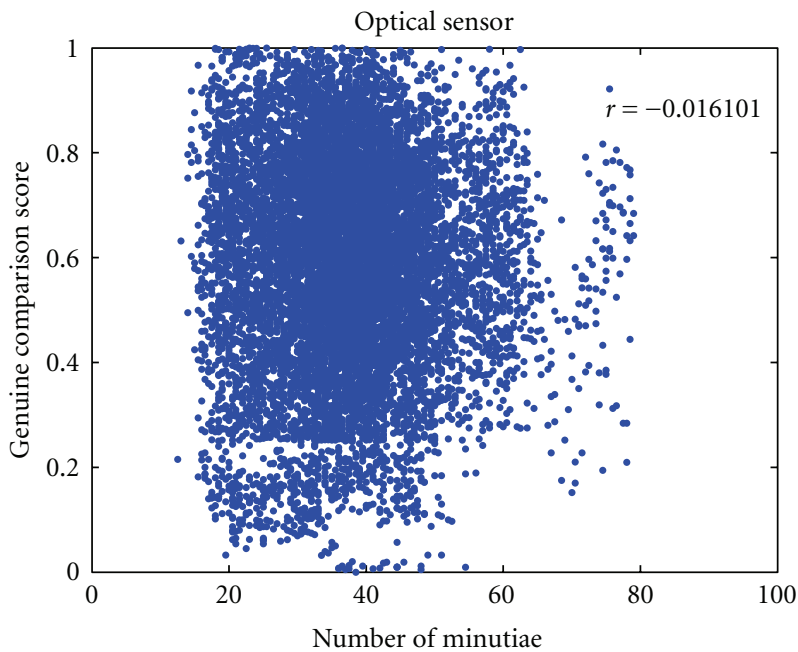

(d)

Figure 6: Genuine comparison scores as a function of the lowest image quality of the two images under test (a,c) and the number of detected minutiae (b, d). However, (a, b) represent the capacitive sensor; (c, d) represent the optical sensor.

Similar to the ratio of a factor of 4 in terms of failures to enroll, the capacitive sensor has an FRR which is also about 4 times larger than the optical sensor for the same FAR.

When images that caused a failure to enroll are not taken into account in the performance evaluation, the error rates improve by almost a factor of two for both sensors (see Table 1). This indicates that the number of failures to enroll, and the number of false nonmatches is about the same for the current database.

A further analysis on the balanced database revealed statistically significant differences in false nonmatch rates between subjects and fingers. When the thresholds for the capacitive and optical sensors were set to individually achieve an FMR of 0.001 between 1.45\% (optical) and 5.05\% (capacitive) of the subjects experienced an FNMR of 0.136 or larger. Moreover, when differences between sensors and fingers are accounted for by setting a separate threshold for each finger index and sensor to obtain an average
FNMR across the population of 0.02 , more than $5 \%$ of the population achieved an FNMR of at least 0.136, which is more than 6 times larger than the population mean, and 4 orders of magnitude larger than expected based on subjectindependent false nonmatch probabilities. Last but not least, $90 \%$ of the population has an FNMR which is smaller than the population average. Said differently, it seems that for this corpus and threshold setting, only $10 \%$ of the population is responsible for the majority of the false nonmatches.

In an attempt to explain high false nonmatch rates for certain individuals, the image quality reported by the template extraction algorithm and the number of extracted minutiae were investigated. These experiments were performed on the balanced database. First, for each combination of sensor, subject, and finger, the FNMR (derived from all 66 comparisons) was correlated with the average image quality and average number of extracted minutiae across all 12 measurements. This correlation thus reflects the 
relation between average properties across all observations of a certain subject and finger, and the average FNMR. No significant first-order relations were found. The resulting Pearson correlations between FNMR and image quality, and between FNMR and the number of minutiae were lower than 0.075 for both sensors.

In a second attempt, the individual comparison scores of all genuine template pairs were correlated with the minimum image quality of the two images under test. This test thus aims at discovering a relation between the comparison score and attributes of the individual images. A scatter plot of comparison score versus image quality for the capacitive sensor is shown in Figure 6(a); the scatter plot for the optical sensor is provided in Figure 6(c). Both the comparison scores and image quality data are normalized to an interval between zero and +1 . The Pearson correlation coefficients $(r)$ are provided in each panel. As can be observed, there is only a weak correlation between image quality and comparison score $(r=0.44$ and 0.42 , for the capacitive and optical sensors, resp.). Figures $6(\mathrm{~b})$ and $6(\mathrm{~d})$ demonstrate the relation between the number of detected minutiae (as mean value of the two templates under test) and the comparison scores. Given the very low Pearson correlation coefficients ( $r=0.12$ and 0.02 ), no relation seems to exist between the number of minutiae and genuine comparison score.

When multiple verification attempts are allowed, the number of false nonmatches reduces by a factor of about 1.7 to 1.8 for two attempts and about 2.2 to 2.4 for three attempts (provided that the FMR is kept constant). This increase in performance is roughly in line with results by others (cf. [4]) and is significantly smaller than what would be expected based on independent probabilities for false nonmatches and false matches for each attempt (cf. (3)-(6)). This observation suggests that the false nonmatch probability for a second or third attempt depends on the outcome of the earlier attempts. If we denote the conditional probability for a false nonmatch during the $N$ th attempt given false nonmatches in all $N-1$ previous attempts by $\psi[\Phi[N], N, N]$, we find the following relation between the overall false nonmatch probability for $N$ and $N-1$ attempts:

$$
\Psi[\Phi[N], N]=\psi[\Phi[N], N, N] \Psi[\Phi[N-1], N-1, N-1] .
$$

If one assumes that the false match rates $\Phi[N]$ are set to a constant value $\Phi$ for every $N$, this results in

$$
\psi[\Phi, N, N]=\frac{\Psi[\Phi, N]}{\Psi[\Phi, N-1, N-1]} .
$$

In other words, the conditional probability for a false nonmatch at trial $N$ given false nonmatches during all earlier attempts can be derived from the ratio of the DET curves for $N$ and $N-1$ attempts. For the current database, in which the relative improvement equals to a factor of approximately 1.75 , this means that the probability of a false nonmatch during the second trial equals approximately 0.57 . Analogously, the conditional probability of a false nonmatch during the third trial, given a false nonmatch during the first and second trial, amounts to approximately 0.75 .
It should be noted that these conditional probabilities describe the average probability for a second or third false nonmatch (i.e., provided that earlier attempts also resulted in a false nonmatch). This result may erroneously be interpreted as an FNMR that depends on the attempt number for a given subject. Most likely, the FNMR rate for a given subject is more or less constant across attempts. The increase in the conditional probability on a system level is presumably caused by an increase in the probability that the current subject is associated with a high (but constant) FNMR, and hence subsequent attempts will (most likely) also have a high probability of a false nonmatch and hence represents a "goat."

\section{Conclusions}

The MCYT fingerprint corpus under test, in combination with a state-of-the-art commercially-available fingerprintmatching algorithm, gives rise to subject-dependent false nonmatch rates if single enrollment and verification measurements are used. This result was observed for a capacitive as well as an optical sensor. From the distribution of false nonmatch rates across subjects, it seems that for a threshold setting resulting in an average false nonmatch rate of 0.02 , a vast majority of $90 \%$ of the population has a probability for a false nonmatch that is smaller than the population average. The average false nonmatch rate seems to be dominated by a small group of subjects that are associated with a disproportionately large number of false nonmatches. When adjusting comparison thresholds as a function of sensor type and finger to result in an average FNMR of 0.02 across the population, at least $5 \%$ of all subjects experienced an FNMR of 0.136 .

In an attempt to predict which images were associated with high false nonmatch rates, fingerprint image quality, the number of detected minutiae, and the genuine comparison scores were compared. Only a weak correlation (Pearson correlation around 0.4 ) was observed between image quality and comparison score, and no significant correlation was found between the number of minutiae and comparison score. This indicates that for the system and corpus under test, these measures cannot reliably indicate images associated with high false nonmatch rates.

The consistency in the false nonmatch probability for certain subjects was expressed as conditional false nonmatch rate. It was observed that for the system under test, the conditional probability of a false nonmatch given 2 earlier attempts amounts to approximately 0.75 . Hence, for the system and fingerprint database under test, the number of verification attempts is best limited to two, and an alternative biometric modality or authentication method should be provided in case a subject experiences two subsequent false nonmatches.

\section{Acknowledgment}

The authors would like to thank the anonymous reviewers and the associate editor for their very helpful comments and suggestions to improve the manuscript. 


\section{References}

[1] P. J. Phillips, W. T. Scruggs, A. J. O’Toole, et al., "FRVT 2006 and ICE 2006: large-scale results," Tech. Rep. IR 7408, NIST National Institute of Standards and Technology, Gaithersburg, Md, USA, 2007.

[2] B. Toth and T. Mansfield, "Latest biometric test resultsperformance, quality and interoperability," Tech. Rep., Deloitte, 2006.

[3] D. Maio, D. Maltoni, R. Cappelli, J. L. Wayman, and A. K. Jain, "IBG comparative biometric testing-round6," Tech. Rep., International Biometric Group, Middlesex, UK, 2006.

[4] T. Mansfield, G. Kelly, D. Chandler, and J. Kane, "Biometric product testing-final report," Tech. Rep., Centre for Mathematics and Scientific Computing, National Physics Laboratory, Middlesex, UK, 2001.

[5] P. J. Phillips, P. J. Flynn, T. Scruggs, et al., "Overview of the face recognition grand challenge," in Proceedings of the IEEE Computer Society Conference on Computer Vision and Pattern Recognition (CVPR '05), vol. 1, pp. 947-954, 2005.

[6] R. Cappelli, D. Maio, D. Maltoni, J. L. Wayman, and A. K. Jain, "Performance evaluation of fingerprint verification systems," IEEE Transactions on Pattern Analysis and Machine Intelligence, vol. 28, no. 1, pp. 3-17, 2006.

[7] Biometric System Laboratory University of Bologna, "FVC2006: the fourth international fingerprint verification competition," 2006, http://bias.csr.unibo.it/fvc2006/default .asp.

[8] G. Doddington, W. Liggett, A. Martin, M. Przybocki, and D. Reynolds, "Sheep, goats, lambs and wolves: a statistical analysis of speaker performance in the NIST 1998 speaker recognition evaluation," in Proceedings of the 5th International Conference on Spoken Language Processing (ICSLP'98), Sydney, Australia, 1998.

[9] N. Yager and T. Dunstone, "Worms, chameleons, phantoms and doves: new additions to the biometrie menagerie," in Proceedings of the IEEE Workshop on Automatic Identification Advanced Technologies (AUTO ID '07), pp. 1-6, Alghero, Italy, 2007.

[10] R. M. Bolle, S. Pankanti, and N. K. Ratha, "Evaluation techniques for biometrics-based authentication systems (FRR)," in Proceedings of the International Conference on Pattern Recognition (ICPR '00), pp. 2831-2837, 2000.

[11] M. Wittman, P. Davis, and P. J. Flynn, "Empirical studies of the existence of the biometric menagerie in the FRGC 2.0 color image corpus," in Proceedings of the IEEE Conference on Computer Vision and Pattern Recognition (CVPR '06), p. 33, 2006.

[12] A. Hicklin, C. Watson, and B. Ulery, "The myth of goats: how many people have fingerprints that are hard to match?" Tech. Rep. IR 7271, NIST National Institute of Standards and Technology, Gaithersburg, Md, USA, 2005.

[13] S. K. Modi and S. J. Elliott, "Impact of image quality on performance: comparison of young and elderly fingerprints," in Proceedings of the 6th International Conference on Recent Advances in Soft Computing (RASC '06), K. Sirlantzis, Ed., pp. 449-454, 2006.

[14] European Parliament and European Council, "Directive 1995/46/EC of the european parliament and of the council of 24 october 1995 on the protection of individuals with regard to the processing of personal data and on the free movement of such data," 1995.
[15] ORC, "Public attitudes toward the uses of biometric identification technologies by government and the private sector. Summary of survey findings, opinion research corporation ORC," 2001.

[16] TNS/TRUSTe, "Consumer attitudes about biometrics in ID documents,” Tech. Rep., TNS/TRUSTe, August 2005.

[17] Logica CMG, "e-identity-european attitudes towards biometrics," Whitepaper, Logica CMG, 2006.

[18] J. Ortega-Garcia, J. Fierrez-Aguilar, D. Simon, et al., "MCYT baseline corpus: a bimodal biometric database," IEE Proceedings: Vision, Image and Signal Processing, vol. 150, no. 6, pp. 395-401, 2003.

[19] F. Alonso-Fernandez, F. Roli, G. L. Marcialis, J. Fierrez, and J. Ortega-Garcia, "Comparison of fingerprint quality measures using an optical and a capacitive sensor," in Proceedings of IEEE Conference on Biometrics: Theory, Applications and Systems (BTAS '07), pp. 1-6, Crystal City, Va, USA, September 2007. 